Aus der Psychiatrischen und Nervenklinik (Direktor: Geheimrat Bonhoeffer) und der Poliklinik für Haut- und Geschlechtskrankheiten (Direktor: Geheimrat Lesser) der Charité in Berlin.

\title{
Untersuchungen über die Spirochäte des Paralytikergehirns.
}

Von Prof. Dr. E. Forster und Prof. Dr. E. Tomasczewski.

Seit der grundlegenden Entdeckung Noguchis, daß in der Hirnrinde der Paralytiker die Spirochaeta pallida vorkommt, ist über ein Jahr verflossen. Die anfänglichen Bedenken, daß es sich dabei um eine Kombination mit Hirnsyphilis oder um eingekapselte, also in gewissem Sinne harmlose Spirochäten handle, wurde sehr bald entkräftet. Schon die Präparate Noguchis bewiesen klar, daß die Spirochäten sich in der Hirnrinde in großen Mengen und, soweit sich das an silberimprägnierten Schnitten beurteilen läßt, in typischer Form vorfanden. Außerdem entsprach das anatomische Bild dieser Präparate durchaus dem der reinen Paralyse. Und endlich war die Zahl der positiven Spirochätenbefunde (48 unter 200 Fällen) sehr viel höher, als es der ja ungemein seltenen Kombination von Lues cerebr und Paralyse entspricht. 
Marinesco und Minea lieferten die erste Bestätigung, indem sie nuit der von Noguchi angewandten Methode unter 26 Fällen von Paralyse in 2 Fällen Spirochäten nachwiesen, von denen allerdings einer ausscheiden muß, da es sich um die seltene Kombination von syphilitischer Meningitis mit Paralyse handelte. Kurz darnach publizierten Marie, Levaditi und Bankowski ihre positiven Befunde.

Alle diese Befunde sind an Leichenmaterial, teils in Schnitt-, teils in Ausstrichpräparaten, teils mit Hilfe der Dunkelfeldbeleuchtung erhoben worden.

Um nachweisen zu können welche Rolle die Spirochaeta pallida in der Pathogenese der Dementia paralytica spielt, wandten wir die Neisser-Pollacksche Hirnpunktion an, weil sie erstens gestattet, Spirochätenuntersuchungen in allen Stadien der Krankheit vorzunehmen, und zweitens ideales Material für Tierimpfungen liefert. Schon am 21. Juni 1913 konnten wir über positive Befunde berichten. Unter vier Punktierten hatten wir in zwei Fällen lebhaft bewegliche Spirochäten gefunden. ${ }^{1}$ ) Fast gleichzeitig teilten Marinesco und Minea mit, daß sie auf dieselbe Weise in einem Fall von juveniler Paralyse Spirochäten nachweisen konnten.

Die Spirochäte war, wie schon erwähnt, von Noguchi in 48 Fällen von $200(24 \%)$ gefunden worden. Marinesco und Minea hatten unter 27 Fällen 3 positive Befunde. Leva diti, Marie und Bankowski gelang der Nachweis in 11 von 41 Fällen, Geber im Verein mit Benedek und Tatar in 15 Fällen zweimal. Außerdem sind vereinzelte positive Befunde noch erwähnt worden von Beriel, Jakob, Levy, Mott, Räcke (Jahnel), Schmorl, Versé.

Unter ihren 41 Fällen haben Levaditi, Marie und Bankowski in einer Serie von 9 Fällen alle Hirnwindungen mit verschiedenen Methoden abgesucht und in acht, die im paralytischen Arfall gestorben waren, Spirochäten gefunden.

Ueber die Frage, ob der Spirochätenbefund in gesetzmäßiger Beziehung zu bestimmten Stadien oder Zustandsbildern der Krankheit steht, konnte durch diese Befunde naturgemäß nichts Sicheres ermittelt werden. Während sich alle anderen Autoren denn auch einer Aeußerung hierzu enthalten, glaubten Levaditi, Marie und Bankowski, wenigstens aus ihren Befunden den Schluß ziehen zu können, daß sich in Fällen, die im paralytischen Anfall sterben, Spirochäten besonders häufig und zahlreich vorfinden.

Unsere eigenen Untersuchungen wurden an 61 Kranken vorgenommen:

Zunächst wurde das rechte und linke Stirnhirn je einmal punktiert; falls keine Spirochäten gefunden wurden, wurde die Punktion jeder Hemisphäre ein- oder zweimal wiederholt.

Von dem Punktionsmaterial wurden kleine Stückchen in physiologischer Kochsalzlösung oder in bei der Punktion mitentleerter Ventrikelflüssigkeit aufgeschwemmt und im Dunkelfelde untersucht. Wir wandten diese Untersuchungsmethode an, weil sie erfahrungsgemä $\beta$ allen anderen Methoden überlegen ist und zugleich ein Urteil über die Beweglichkeit d. h. eine Lebensäußerung des Krankheitserregers gestattet.

In 61 untersuchten Fällen fanden wir Spirochäten $27 \mathrm{mal}$, d. h. in $44 \%$. Elfmal fanden wir zahlreiche Spirochäten, zum Teil so zahlreich wie in den spirochätenreichen Krankheitsherden der primären, sekundären und kongenitalen Syphilis. In 16 Fällen mußten wir aber erst viele Präparate längere Zeit (1-2 Stunden) durchsuchen, bis wir eine oder einige wenige Spirochäten auffanden.

Unsere Prozenzahl positiver Spirochätenbefunde ist die höchste bisher gefundene und ist fast doppelt so groß als die Noguchis. Dabei haben wir nur kleinste Teilchen der Hirnrinde untersucht, allerdings mit der besten Methode und am Lebenden. Berücksichtigt man, da 3 wir in vielen Fällen nur im Punktionsmaterial einer Hemisphäre Spirochäten fanden und zuweilen nur in einem von mehreren Hirnzylindern eines Bohrloches, so ist die Annahme erlaubt, daß auch in den negativen Fällen der,Spirochätennachweis in Hirnzylindern von anderen Stellen schließlich gelungen wäre; denn in unserem Material hat sich kein Anhaltspunkt dafür ergeben, daß die Zeit zwischen Infektion und Ausbruch der Paralyse oder die Dauer der paralytischen Krankheitserscheinungen zum Spirochätenbefunde in Beziehung steht. Das Gleiche gilt für das klinische Zustandsbild. So fanden wir in einfach dementen Formen manchmal zahlreiche Spirochäten, in Fällen mit paralytischen Anfällen und expansiven Größenideen fehlten sie öfter. Sogar in einem Fall, den wir noch vor völligem Ab-

1) D. m. W. 1913 Nr. 26 S. 1237. klingen des paralytischen Anfalles punktierten, fanden wir keine Spirochäten.

Daß Marie, Levaditi und Bankowski bei 8 im paralytischen Anfall Verstorbenen stets zahlreiche Spirochäten fanden, erklärt sich vielleicht besser aus der sorgfältigen Untersuchung sehr zahlreicher Rindenpartien als aus der speziellen Todesursache.

Vielleicht verdient noch Erwähnung, daß unter unseren drei Fällen von juveniler Paralyse zweimal zahlreiche Parasiten gefunden wurden.

Die Spirochäten zeigten ausnahmslos den Typus der Spirochaeta pallida; denn sie hatten ein schwaches Lichtbrechungsvermögen, zahlreiche enge, steile Windungen, die nach den Enden zu an Höhe abnahmen und, wie sich wiederholt feststellen ließ, in einen feinen Endfaden ausliefen, und charakteristische, meist sehr lebhafte Bewegungen: Drehungen um die Längsachse, zappelnde und peitschende Bewegungen ohne nennenswerte Ortsveränderungen. Einige Male sahen wir 2 bzw. 3 Spirochäten im Verband, einmal ein Büschel von Spirochäten. Ihr tinktorielles Verhalten war eigentümlich. In drei Fällen färbten wir Präparate, die bei Dunkelfeldbeleuchtung zahlreiche Spirochäten erkennen ließen, in der üblichen Weise mit Giemsalösung. Doch konnten wir in den gefärbten Präparaten keine Spirochäten wiederfinden. Wohl aber gelang das sehr leicht, als wir dieselben Präparate in der zuerst von Fontana empfohlenen, später von Tribondeau modifizierten Weise färbten. Die Methode besteht darin, daß man die Ausstriche in Formol fixiert, dann mit einer ammoniakalischen Silberlösung beizt und mit Tannin nachbehandelt. Die Spirochäten erscheinen als dunkelbraune resp. schwarze Gebilde und lassen sich leicht auffinden. In der Literatur haben wir keinen Hinweis auf dies eigenartige tinktorielle Verhalten gefunden.

Tierversuche mit Hirnsubstanz frischer Paralytikerleichen haben Landsteiner und Pötzl schon 1908 angestellt. Sie impften Affen und hatten zwar, ,ein Verdacht erweckendes, aber kein eindeutiges Resultat". Ueber sicher positive Resultate hat zuerst Noguchi berichtet.

Als Impfmaterial verwandte er Hirnrinde von 6 Paralysen, die kurze Zeit post mortem zur Sektion gekommen waren. Nur in einen dieser Fälle ließen sich Spirochäten nachweisen. Von jedem Fall wurden je 6 Kaninchenböcke in die Hoden geimpft. Von den 30 Tieren, die mit spirochätenfreiem Material inokuliert waren, erkrankte keines. Von den 6 Kaninchen, deren Impfmaterial Spirochäten enthielt, erkrankten 2 Tiere, das eine nach 87, das andere nach 102 Tagen; die zweite Generation dieses Stammes brachte nach Verimpfung auf die Hoden von 4 Kaninchen in einem derselben ziemlich kleine und indurierte Läsionen hervor nach einer Inkubationsperiode von drei Monaten. Es bildeten sich kleine Knötchen, die typische Spirochäten enthielten.

Ferner haben Uhlenhuth und Mulzer ein sicher positives Impfresultat erhalten.

Sie verimpften Hirnrinde von 5 Fällen von Paralyse kurze Zeit post mortem in die Hoden von Kanunchen. Bei einem Tier entwickelte sich nach 50 Tagen ein kleines Knötchen, das sehr zahlreiche feinste Spirochäten enthielt.

Kurz nach Noguchi und schon vor Uhlenhuth und Mulzer hat Berger über positive Impfresultate berichtet.

Er impfte Ende März und Anfang April 1913 mit Punktionsmaterial von 20 Paralysen 20 Kaninchen. Bei drei Tieren beobachtete er nach 69 , 81 und 110 Tagen kleinste Knötchen. In Schnittpräparaten fand Berger in der Umgebung der Infiltrate nach langem Suchen mehrere Spirochäten, obwohl in zwei dieser Fälle, die auch im Dunkelfeld untersucht worden waren, Spirochäten nicht nachgewiesen werden konnten.

Wie schon Uhlenhuth und Mulzer hervorheben, sind die Bergerschen Befunde sehr auffallend, da die Produkte der experimentellen Kaninchensyphilis sehr spirochätenreich zu sein pflegen.

Unsere eigenen Tierversuche, die seit Anfang Juni 1913 im Gange sind, sind bisher sämtlich negativ ausgefallen.

Im ganzen haben wir mit dem Punktionsmaterial von 53 Fällen 60 Kaninchenböcke in die Hoden geimpft; von diesen Tieren starben 5 schon 5, 16, 25, 42 und 49 Tage post infectionem, 15 Tiere konnten wir über zwei Monate, 40 über vier Monate beobachten, ohne daß irgendwelche örtlichen oder allgemeinen Krankheitserscheinungen auftraten. Außerdem impften wir 13 Affen in die Lider, und zwar ausschließlich mit spirochätenreichem Punktionsmaterial. Von den Affen starb einer schon 27 Tage post infectionem, 5 starben $61,82,86,88$ und 90 Tage nach der 
Impfung; 7 sind über vier Monate in Beobachtung. Bei 5 entwickelten sich $4-5$ Wochen post infectionem kleine Knötchen in der Lidhaut, in deren Reizserum sich aber trotz wiederholter Untersuchung im Dunkelfeld keine Spirochäten nachweisen ließen. Im weiteren Verlauf der Beobachtung bildeten sich diese Knötchen vollkommen zurück. Intrazerebrale Impfungen blieben bisher ebenfalls erfolglos.

Das negative Ergebnis unserer.Tierversuche ist gewiß sehr auffallend, denn das Impfmaterial war lebensfrisch, enthielt in 25 Fällen mikroskopisch Spirochäten, darunter $10 \mathrm{mal}$ zahlreiche resp. sehr zahlreiche, lebhaft bewegliche. Außerdem erfolgte die Impfung in der Hälfte der Fälle fast unmittelbar 1lach der Punktion, und auch in den übrigen Fällen, in denen das Impfmaterial vor der Inokulation in feuchter Kammer oder in warmer physiologischer Kochsalzlösung aufbewahrt worden war, erfolgte die Impfung durchschnittlich nach etwa $1 / 4$ bis $1 / 2$ Stunde. Fast in jedem Falle wurden mehrere Hirnzylinder verimpft; besonders reichlich war das Impfmaterial bei den Affeninokulationen. Und endlich betrug die Beobachtungsdauer bei 40 Kaninchen und 7 Affen über vier Monate. Für die Beurteilung dieser negativen Impfresultate sei daran erinnert, daß Inokulationen mit den Krankheitsherden der tertiären Syphilis, in denen fast nie Spirochäten zu finden sind, bei Affen in der Regel positiv ausfallen. ${ }^{1}$ )

Durch die Spirochätenbefunde wurde das Krankheitsbild der Paralyse, das sich in so typischer Weise von der Hirnsyphilis unterscheidet, womöglich noch unerklärlicher. Durch das Resultat unserer Untersuchungen ist nun eine experimentelle Grundlage für eine Deutung geschaffen; denn unsere Experimente legen die Annahme nahe, daß die Spirochäte in der Hirnrinde der Paralyse von der gewöhnlichen Syphilisspirochäte biologisch abweicht. So verliert die lange Inkubationszeit, die Eigenart des pathologisch-anatomischen Befundes und der typische Verlauf etwas von dem Rätselhaften. Damit erhält die schon früher von Erb supponierte Ansicht von der biologischen Veränderung der Spirochäte der Paralyse ihre Bestätigung.

Dieser Ansicht widersprechen die positiven Resultate von Noguchi, Berger, sowie von Uhlenhuth und Mulzer keineswegs. In ihren Versuchen ist nämlich Folgendes auffallend: Zunächst die geringe Zahl der Impferfolge, ferner die Geringfügigkeit der Erscheinungen und endlich die lange Inkubationsdauer, die nur bei Uhlenhuth und Mulzer annähernd normal war. Wir haben also auch in diesen Versuchen Anhaltspunkte für eine biologische Veränderung der Spirochäten in der Hirnrinde der Paralyse. Gestützt wird ferner unsere Anmahme durch weitere negative Resultate anderer Autoren. So berichtet E. Hoffmann, daß auch an der Bonner psychiatrischen Klinik Impfungen auf Kaninchen mit Leichenmaterial keinen Erfolg gehabt haben. Ferner erwähnt Nonne, daß er in einem Falle ein durch Trepanation gewonnenes kleines Stück aus der Rinde des Frontalhirns eines schweren Falles von Paralyse auf Kaninchen ohne Erfolg geimpft hat. Auch Ceraditi teilte uns schriftlich mit, daß er bisher keine positiven Impfresultate erzielt habe. Endlich haben noch Marinesco und Minea, sowie Gerber und Benedek Kaninchenimpfungen mit spirochätenhaltiger Hirnrinde von Paralyse vorgenommen. Ueber das Resultat haben aber diese Autoren bisher nichts mitgeteilt, trotzdem seit der Impfung schon über sechs Monate verflossen sind. ${ }^{2}$ )

Daß die Paralyse durch eine biologisch veränderte Syphilisspirochäte verursacht wird, kann nach alledem als sehr wahrscheinlich gelten. Freilich ist noclı unerklärt, wann, wo und wie diese Veränderung zustande kommt.

Literatur: Berger, M. m. W. 1913 Nr. 35 S. 1921. - Beriel, Neurol. Zbl. 1914 Nr. 1 S. 21. - Fontana, Pathologia 5. 1913. Nr. 109. - Forster u. Toinasczewski, Sitzung d. Psychiatr. V. i. Berlin, 21. Vl. 1913. Allg. Zschr. f. Psych. 70. 1913. S. 855 . - D. m. W. 1913 S. 1237. - Geber u. Benedek, W. kl. W. 1913 Nr. 40 S. 1624. - Geber, Benedek u. Tatar, W. kl. W. 1913 Nr. 38 S. 1491 E. Hof 1913. Jakob. D. W. W. 1914 Nr. 2 S. 100 . Landsteiner, Zbl. Bakt 41. 1908. S. 785. - Levaditi, Marie et Bankowski, Bullet. de la soc. franc. Bakt. 41. 1008. S. 785. Leve 1913 Nr. 27 . 377. Marie, Levaditi et Bankowski, Compt. rend. hebdom. des séanc. de la soc. de biologie 74. 1913. S. 794 und 1009. - Marinesco et Minea, Compt. rend. des

1) Vgl. die Zusammenstellung hierüber in Tomasczewski, Arch. f. Derm. u. Syph. 113. 1912. S. 1133. _ ${ }^{2}$ ) Positive Impfresultate mit Blut von Paralysen haben Graves und Levaditi, mit Liquor cerebrospinalis Mattauschek und Volk mitgeteilt. sẻanc. de la soc. de biologie 75. 1913. S. 231. Bulletin de l'acadèmie de mëdecine 4 1913. Revue neurolog $1913 \mathrm{Nr}$ 21 S. 231 . Mott. Internat. med Kongr. London 1913. Nichol. Mongr., London 1913. - Nichols and Hough, Journ. americ. med. Assoc. 1913 Nr. 2 S. 120

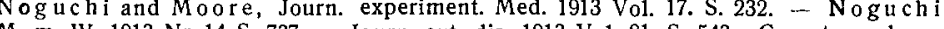
M. m. W. 1913 Nr. 14 S. 737. - Journ. cut. dis. 1913 Vol. 81, S. 543. Compt. rend. soc. de biol. 74. 1913. S. 349. Journ. americ. med. Assoc. 1913 Vol. 61 S. 85. B. kl. W. 1913 Nr. 41 S. 1884. D. m. W. 1913 Nr. 33 S. 1604. - Nonne, D. Zschr. f. Nervernhlk. 49. 1913 S. 385. - Ra ecke, M. m. W. 1914 Nr. 5 S, 272. - Schmor 1, M. m. W, 1913 Nr. 30 S. 1685 . - Tribondeau, Bull. de 1a soc. franç. de dermat. 1912 p. 474 . Uhlenhuth u. Mulzer, B. kl. W. 1913 Nr. 44 S. 2031. - Versé, M. m. W. 1913 Nr. 44 S. 2446. 